\title{
COMHUMANITAS
}

Revista ComHumanitas, ISSN: 1390-776X

Vol. 11, núm. 3 (2020), Septiembre - Diciembre 2020

DOI: https://doi.org/10.31207/rch.v11i3.274

\section{Ludificación informativa en tiempos de pandemia: newsgames y la Covid-19i}

\section{Informative's Gamification in the Times of Pandemic: Newsgames and Covid-19}

\section{Gamificação de informações em tempos de pandemia: newsgames e Covid-19}

\author{
Nuria Navarro-Sierra ${ }^{1}$ \\ Universidad Rey Juan Carlos (España) \\ nuria.navarro.sierra @urjc.es \\ Raquel Quevedo-Redondo ${ }^{2}$ \\ Universidad de Valladolid (España) \\ raquel.quevedo.redondo@uva.es
}

Fecha de recepción: 30 de noviembre de 2020

Fecha de recepción evaluador: 13 de diciembre de 2020

Fecha de recepción corrección: 21 de diciembre de 2020

\footnotetext{
${ }^{1}$ Doctora con mención internacional en Comunicación audiovisual por la Universidad Complutense de Madrid, donde inició su formación docente a través de la beca FPU del Ministerio de Educación en España. Profesora ayudante doctora en los dobles grados de Periodismo, Publicidad y Comunicación Audiovisual de la Universidad Rey Juan Carlos. Cuenta con la acreditación de profesora contratada doctora. ORCID: https://orcid.org/0000-0002-1431-1534

${ }^{2}$ Doctora en Periodismo por la Universidad de Valladolid, donde imparte clases que compagina con docencia en la Universidad Europea Miguel de Cervantes y en la Universidad Permanente Millán Santos. Actualmente cuenta con la acreditación de profesora contratada doctora, que alcanzó tras un periodo formativo como beneficiaria de una beca FPU del Ministerio de Educación en España. ORCID: https://orcid.org/0000-0002-6219-3237
} 


\title{
Resumen
}

La situación de excepcionalidad producida por la covid-19 propicia un escenario comunicativo complejo en el que la cobertura informativa de la pandemia ha planteado cambios sobre las dinámicas habituales. Los medios de comunicación se han enfrentado a nuevos desafíos para reducir la incertidumbre y satisfacer los procesos de búsqueda de información a través de un amplio número de formatos. En este contexto, el objetivo de la presente investigación se centra en localizar y analizar los newsgames que han abordado el devenir noticioso con la finalidad de ofrecer un distinto enfoque y contenido a los usuarios. Esa lista la integran tres juegos: Factitious 2020! Pandemic Edition (Augame Studio, 2017-20), People of the Pandemic (Wu \& Osserman, 2020) y Covid-19 Trivia (Mindtoggle, 2020). El trabajo de campo se apoya en la metodología cualitativa para someter a análisis tanto las propuestas informativas como la estrategia discursiva que plantean los juegos digitales a partir de los rasgos definidos por Janet H. Murray (1999): actuación, inmersión y transformación. Lo limitado de la muestra de análisis permite afirmar que el uso de recursos lúdicos no ha sido aprovechado por los medios de comunicación para dar a conocer algunos de los rasgos más complejos de la pandemia y sacar partido a una fórmula que, cuando se utiliza, facilita la comprensión a determinados segmentos de público.

Palabras clave: covid-19; newsgames; medios interactivos; ludificación.

\begin{abstract}
The exceptional situation produced by covid-19 fosters a complex communicative scenario in which news coverage has seen its usual dynamics modified. The media have faced the challenge of reducing uncertainty while increasing the search for information and, for this, a wide number of formats have been used. Therefore, the objective of this research is to locate and analyze the newsgames that have addressed the situation of the pandemic in order to offer other types of information to their users: Factitious 2020! Pandemic Edition (Augame Studio, 2017-20), People of the Pandemic (Wu \& Osserman, 2020) and Covid-19 Trivia (Mindtoggle, 2020). The analysis of its informative objectives is based on a qualitative analysis methodology that places its informative proposals together with its discursive strategy as a digital game based on the features defined by Janet H. Murray (1999): acting, immersion and transformation. The limitation of the sample suggests that the use of playful formats has not been used by the media to present some of the more complex features of this situation under a formula that, when used, could have facilitated the understanding of the public.
\end{abstract}

Keywords: Covid-19; Newsgames; Interactive Media; Gamification.

\section{Resumo}

A situação excepcional produzida por covid-19 fomenta um cenário comunicativo complexo, no qual a cobertura noticiosa viu sua dinâmica usual modificada. Os meios de comunicação têm enfrentado o desafio de reduzir as incertezas e, ao mesmo tempo, 
aumentar a busca por informações e, para isso, diversos formatos têm sido utilizados. Portanto, o objetivo desta pesquisa é localizar e analisar os newsgames que abordaram a situação da pandemia, a fim de oferecer outros tipos de informação aos seus usuários: Factitious 2020! Pandemic Edition (Augame Studio, 2017-20), People of the Pandemic (Wu \& Osserman, 2020) e Covid-19 Trivia (Mindtoggle, 2020). A análise de seus objetivos informativos baseia-se em uma metodologia de análise qualitativa que alia suas propostas informativas à sua estratégia discursiva como um jogo digital a partir das características definidas por Janet H. Murray: atuação, imersão e transformação. A limitação da amostra sugere que o uso de formatos lúdicos não tem sido utilizado pela mídia para apresentar algumas das características mais complexas dessa situação sob uma fórmula que, quando utilizada, poderia ter facilitado o entendimento do público.

Palavras-chave: covid-19; newsgames; mídia interativa; gamificação.

\section{Introducción}

La complejidad y el alcance de la crisis producida por la pandemia de la covid-19 ha generado una situación inédita que ha tenido profundas repercusiones en diversas esferas de la sociedad contemporánea. Entre otros efectos, se ha incrementado el interés por diagnosticar y comprender la influencia del coronavirus en ámbitos propios de las ciencias de la salud, las ciencias sociales y las humanidades. En este contexto, se entiende que en el terreno de la investigación académica haya tenido lugar la aparición de un ingente número de publicaciones sobre las diferentes aristas del nuevo campo de estudio, con la consiguiente puesta a prueba de la capacidad del sistema para producir, revisar y publicar una enorme cantidad de conocimiento producido en un periodo de tiempo muy breve (Kupferschmidt, 2020). La fertilidad de este interés refleja la multidisciplinariedad del fenómeno, su relevancia científica, social y académica e, incluso, el afán por aprovechar su popularidad e impacto. De esta forma, el aumento de estudios con la covid19 como eje temático hizo que se publicaran entre 500 y 1000 artículos sólo en los meses de abril y mayo, llevando a identificar la proliferación de estos trabajos como parte de "un fenómeno clásico bibliométrico de crecimiento exponencial" (Torres-Salinas, 2020).

La realidad expuesta implica que los efectos de la pandemia se hayan analizado bajo un prisma heterogéneo que, sólo en el ámbito de la Comunicación Social, propone una ramificación temática increíblemente extensa como consecuencia de una cobertura informativa casi hegemónica durante los meses de mayor intensidad clínica del virus y el elevado número de noticias que han ofrecido los mass media a propósito del mismo. A este respecto cabe recordar que, tal y como indica Casero-Ripollés (2020), "en situaciones críticas de alta complejidad y riesgo para la vida humana como el brote del nuevo coronavirus, los ciudadanos sitúan la búsqueda de información y el seguimiento de las noticias como actividades clave".

La infoxicación generada tanto en medios de comunicación como en redes sociales ha supuesto un conjunto de retos para políticos, portavoces sanitarios y 
científicos a la hora de definir la amenaza que entraña la covid-19 para la población y de "cambios que han llegado para quedarse" (Diviu-Miñarro \& Cortiñas-Rovira, 2020), ya que el conflicto entre la implementación de medidas impopulares y las tensiones políticas, económicas y sociales detectables en los diferentes países ha dificultado la gestión institucional de la comunicación (Romero-Rodríguez \& Rodríguez-Hidalgo, 2019; Vicente-Fernández, et al., 2020). A la cobertura informativa tradicional, además, se ha sumado la incorporación de nuevas narrativas, medios o plataformas como apps, videos en $360^{\circ}$ y contenidos en TikTok (Gil-Torres, et al., 2020; Sidorenko-Bautista, Herranz \& Cantero, 2020; Ballesteros Herencia, 2020) que han mostrado todo su potencial para diferenciarse de los soportes clásicos y despertar el interés de un público abrumado por los patrones sensacionalistas y el sesgo ideológico de los medios convencionales (Masip, et al., 2020). Del nuevo ecosistema también han formado parte iniciativas infográficas e interactivas que perseguían aligerar la carga informativa y reducir el grado de abstracción, ambigüedad y confusión imperante (Andreu-Sánchez \& Martín-Pascual, 2020), y si bien la apuesta por la divulgación científica a través de elementos visuales ha servido para minar la incertidumbre y mejorar la toma de decisiones para proteger la salud individual y colectiva (Casino, 2019), la reflexión sobre este tipo de fórmulas de presentación no ha sido demasiado prolífica (Andreu-Sánchez \& Martín-Pascual, 2020, p. 8).

La atracción suscitada por infografías como How Coronavirus Mutates and Spreads (edición digital The New York Times, 30 de abril de 2020) o Un salón, un bar y una clase: así contagia el coronavirus en el aire (edición digital El País, 24 de octubre de 2020) constatan el interés, alcance y viabilidad de los nuevos formatos narrativos del periodismo digital en un contexto de sobreexposición informativa como el que aborda esta investigación (Fundamentium, 2020), pero, sobre todo, insta a que la comunidad científica dedique mayor atención a las propuestas informativas menos tradicionales, ya sean de carácter pedagógico o de otra categoría.

La presente propuesta parte de la última consideración para definir el principal objetivo de la investigación: analizar las propuestas informativas de carácer lúdico e interactivo que se han desarrollado en relación con la covid-19 para descubrir su contribución al conocimiento en el contexto de la pandemia. Una decantación informativa que se concreta en los denominados newsgames o juegos digitales inspirados en temas de actualidad (Gómez \& Cabeza, 2016), con la intención no sólo de informar o concienciar a la población, sino también de alimentar el debate público (Sicart, 2008). Así, este trabajo analiza las diferentes iniciativas que se han desarrollado a lo largo del año 2020, sin perder de vista cómo se ha participado en el debate informativo y social en torno a la pandemia. Para ello, se proponen las siguientes preguntas de investigación:

○ $\mathrm{PI}_{1}$. ¿Cuál ha sido la participación informativa de los newsgames en relación con la crisis de la covid-19? La respuesta a esta pregunta parte de la localización de los juegos digitales que han contribuido a la generación de conocimiento para identificar sus características más significativas tanto en el campo de la información como en el del entretenimiento. 
- $\mathrm{PI}_{2}$. ¿Cuáles han sido los rasgos del discurso de los newsgames sobre la covid-19, ya sea como recurso informativo o como propuesta lúdica? A partir de esta pregunta se espera profundizar en la propuesta de discursos que ofrecen los nuevos formatos de gamificación y en su hipotética eficacia.

\section{Marco Referencial}

La fundamentación teórica de esta investigación se apoya en la intersección entre la extensa cobertura periodística de la covid-19 y las propuestas informativas de carácter lúdico-interactivo desarrolladas sobre este mismo eje temático en 2020. Un marco que, a pesar de su ajustado arco temporal, se asienta sobre una tarea de revisión bibliográfica que ha superado con creces la de la producción científica en áreas populares de otras disciplinas científicas.

La investigación en comunicación sobre la pandemia comprende análisis clásicos sobre la producción de noticias en prensa escrita (Monjas Eleta, Rodríguez Holguín \& Gil-Torres, 2020) y en ediciones digitales de los diarios (Meneses-Fernández \& SantanaHernández, 2020); disertaciones sobre la adaptación de contenido para los oyentes de radio (Rodero, 2020) o espectadores de la televisión pública (Villena-Alarcón \& Caballero-Galeote, 2020), y evaluaciones del uso dado a las herramientas que se han incorporado en los últimos años a los flujos de comunicación (Salles \& Dall'orto, 2020).

Sobre el análisis de newsgames relacionados con el coronavirus, el vacío académico conlleva prestar mayor atención que al resto de esferas citadas. Por este motivo, la investigación que ocupa estas páginas fundamenta en su carácter innovador la necesidad de observar la construcción informativa de la covid-19 propuesta por los juegos digitales, tomando como referencia una conocida afirmación de Marshall McLuhan: los juegos "son extensiones de nuestro ser, no individual sino social, y debería haber quedado bien claro que son medios de comunicación" (1996, p. 253). Esta apreciación se acentúa en el ámbito de los denominados "juegos serios" (Gómez, 2014), con lo que la aparición de los newsgames permite hablar de aquellos productos que se localizan "en la intersección entre el periodismo y los videojuegos" (Bogost, et al, 2010, p. 13). Un cruce de caminos que se desarrolla en el contexto de la convergencia mediática de la primera década de este siglo (Vobic, Dvorsak \& Vtic, 2014, pp. 123-124) y el amplio conjunto de rasgos que definen al videojuego como uno de los nuevos medios de comunicación de masas (Dovey \& Kennedy, 2006, pp. 84-103).

September 12th (Powerful Robots, 2003) concretó el concepto de "newsgame" brindado por su creador, Gonzalo Frasca, para describir un género emergente, basado en noticias de actualidad y distinto de las propuestas convencionales, a tenor de que "tradicionalmente, los videojuegos se han centrado en la fantasía por encima de la realidad", pese a ser "excelentes herramientas para mejorar nuestra comprensión del mundo". Esta conceptualización, débil en muchos aspectos desde el punto de vista teórico, refleja en todo caso un rasgo fundamental del contenido a analizar: la relación 
que se establece entre la temática del videojuego y la actualidad noticiosa en una lógica de fusión entre la simulación lúdica y los aspectos actuales que son relevantes para la sociedad.

En este estudio se comprueba que los newsgames son un concepto que se construye a partir de una combinación de los códigos propios de los Game Studies tanto en lo narrativo como en su interacción con el usuario, pero también que "realmente no se tiene del todo clara una definición precisa" (Plewe \& Fürsich, 2018, p. 3). Quizá esa cierta ambigüedad detectada en la revisión del estado de la cuestión se deba a que la presencia de recursos lúdicos en el panorama mediático no resulta cuantitativamente significativa, no obstante, es justo subrayar que sí lo es en términos cualitativos, pues algunos medios de comunicación han apostado con éxito por ellos en los últimos veinte años, como prueba el newsgame más popular de la edición digital del New York Times durante el año 2013: How Y'all, Youse and You Guys Talk (Meier, 2018). Del mismo modo y a pesar de la falta de concreción sobre el concepto que nos ocupa, existe un interés académico creciente y que se traduce en una producción académica cada vez más consolidada en revistas como Convergence: The International Journal of Research into New Media Technologies, donde se publicó el monográficos "Playful approaches to news engagement" (2020). Esta percepción sobre el potencial de los videojuegos en el ámbito informativo no es, por tanto, una tendencia residual... La comparten múltiples profesionales entre los que cabe citar a Nikki Usher y su monografía sobre el New York Times a propósito de la interactividad:

Los 'videojuegos' han tenido un impacto en la redacción. Los juegos no solo han generado un tráfico web masivo, sino que habían llevado la serie [de serious games] de Richtel "Driven to Distraction" — conducir con teléfonos móviles - a un nivel completamente nuevo. Desde la comodidad de su computadora, puede probar una simulación que le permite enviar mensajes de texto y conducir de forma segura. Uno de los juegos comprueba su capacidad para conducir entre las cabinas de peaje de las autopistas mientras responde mensajes de texto sobre su postre favorito desde un teléfono celular simulado. El subtexto de la declaración de Richtel a la sala de redacción fue bastante poderoso: el periodismo tradicional es ahora solo un elemento para contar historias. La interactividad permite a los periodistas crear una experiencia completamente nueva para los lectores (Usher, 2014).

A pesar de que es pronto para determinar el acierto de Matt Richtel a la hora de evaluar el futuro del interactivo o de la incorporación de los juegos en el discurso de los medios de comunicación tradicionales, resulta evidente el anhelo por constituirlos como una punta de lanza del actual periodismo digital. Una situación que se inscribe en una estrategia innovadora en la pugna por la atención dispersa de los internautas (Plewe \& Fürsich, 2018, p. 12) y la búsqueda de un mayor nivel de consulta y fidelización de los usuarios (Ferrer \& Karlsson, 2016, p. 5) a través de la integración en los contenidos informativos de los videojuegos, la realidad virtual y los vídeos $360^{\circ}$ (Mañas-Viniegra, Zamith \& López-Cepeda, 2019, p. 154).

En síntesis, aunque no cabe duda de que la incorporación de los newsgames al discurso informativo de los mass media aporta una nueva capa a la tensión que soportan 
los estándares del "periodismo profesional" en el marco narrativo de la crisis de la covid19, también genera nuevas oportunidades para los medios y un desafío que algunos investigadores interpretan como una motivación añadida:

Si los newsgames y otros enfoques lúdicos del periodismo van a asumir una posición más dominante dentro de la distribución de noticias convencional [...], deberían sacar a los periodistas y creadores de juegos de sus zonas de confort, exigiéndoles que desafíen las ortodoxias percibidas, los supuestos filosóficos y editoriales, la ética y las practicas digitales (Ferrer, et al., 2020, p. 10).

Así, ante un escenario rupturista con los esquemas tradicionales, cabe preguntarse cuál es el nivel de ludificación que el periodismo puede aceptar y, de manera más exacta, cuál debe ser su graduación dentro del discurso informativo.

\section{Metodología}

\section{Muestra y procedimiento}

La localización de la muestra seleccionada para esta investigación se realizó a partir de motores de búsqueda que han respondido a la combinación de palabras clave en español e inglés (newsgames, juegos digitales, videojuegos, covid-19, coronavirus, 2019nCoV y SARS-CoV-2). Además, se revisaron bases de datos y catálogos como los propios de Serious Games Classification, Newsgames Directory, Journalism Games o Games 4 Change. Los resultados permitieron apoyar el trabajo de campo en tres propuestas que suponen todos los newsgames disponibles sobre la materia que nos ocupa en el mes de noviembre de 2020, y que se presentan de manera sintética en la tabla 1.

Tabla 1. Muestra de newsgames sobre la covid-19

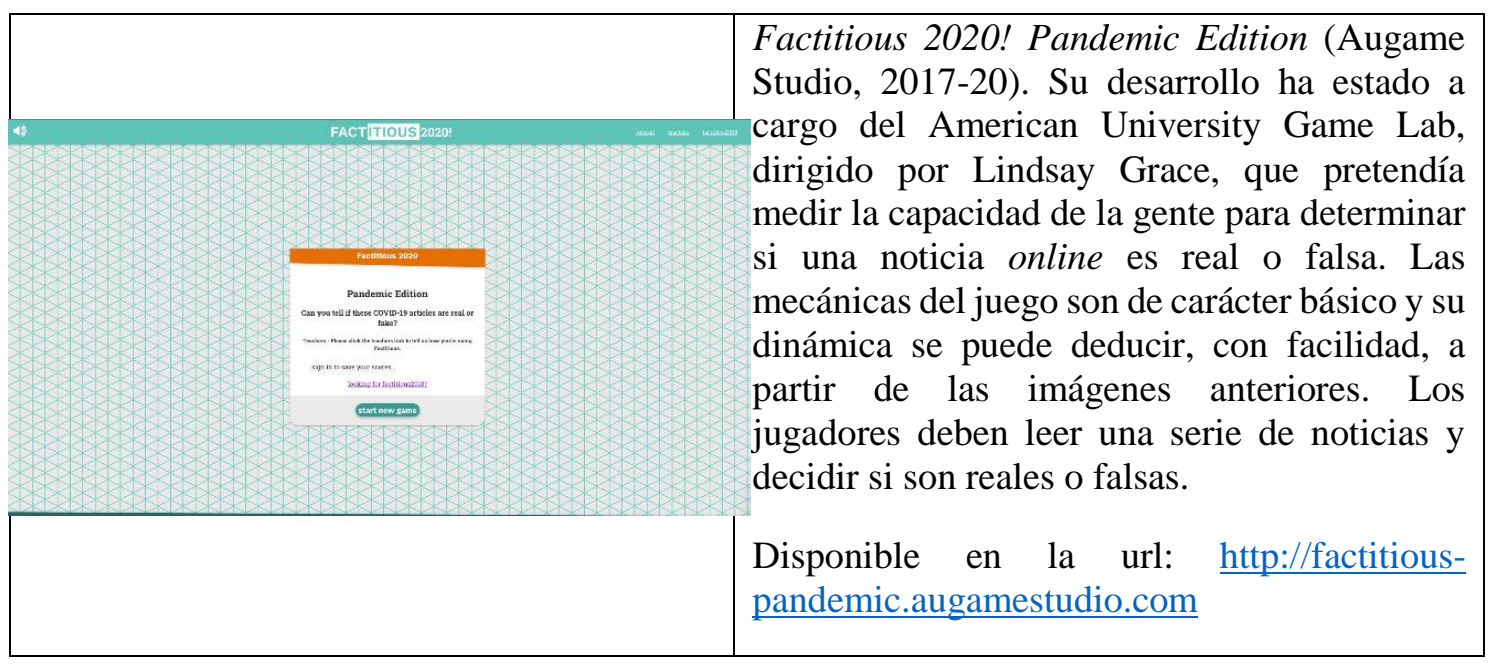




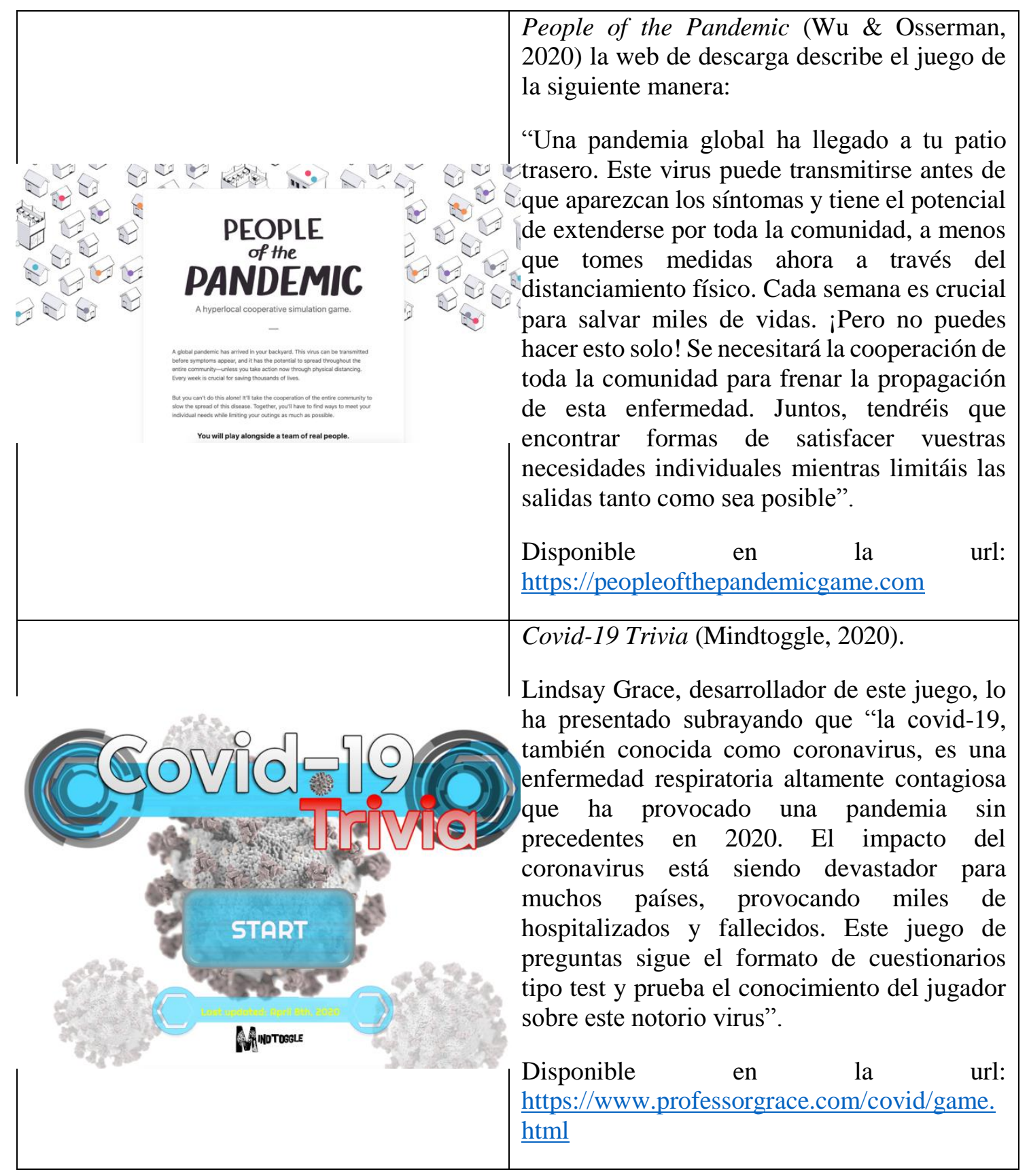

Elaboración propia.

Ciertamente, después de la primera labor de muestreo se localizaron otras iniciativas de interés como VRS Home Mission (Omnium Lab, 2020), pero esta clase de juegos responde más a una lógica pedagógica generalista que a la línea de información especializada característica de los newsgames. Por esta razón, no fueron incluidos en el trabajo de campo.

\section{Propuesta metodológica}

Los objetivos planteados en cada juego de la muestra permitieron definir mejor la estrategia metodológica, enfocada a identificar el discurso de los newsgames y los recursos de alfabetización informativa para promover el conocimiento y la concienciación sobre la covid-19. Por otra parte, la naturaleza de los newsgames como 
ramificación de los formatos inherentes al Periodismo Digital y a los juegos serios (Gómez-García \& Carrillo-Vera, 2020) animó a las investigadoras a emplear un método de análisis textual desarrollado con éxito en otras investigaciones (Carr, 2009; Plewe \& Fürsich, 2018), a fin de focalizar el interés tanto en las estrategias narrativas e informativas como en las reglas y mecánicas que cada juego plantea.

De esta forma, esta propuesta se subordina a tres niveles concretos de análisis que han seguido un procedimiento inductivo (definición de los códigos y, posteriormente, realización del proceso de codificación). El primero de ellos aborda la construcción narrativa e informativa de los juegos digitales y pretende identificar los rasgos que cumplen los newsgames entendidos como productos informativos y su adaptación a patrones básicos de la actividad periodística (García-Ortega \& García-Avilés, 2018). En segundo lugar, se atiende a su consideración como juego en cuanto a su "forma abstracta de contar una historia que se parece al mundo de la experiencia común, pero que lo reduce para aumentar el interés" (Murray, 1999, p. 155). Finalmente, se tienen en cuenta los principales rasgos que se han definido como propios de este formato: inmersión, actuación y transformación (Murray, 1999).

\section{Hallazgos}

La interpretación de los códigos asociados a cada juego de la muestra permite una construcción acorde a los diferentes niveles de la metodología. Así, se ofrecen resultados sobre la articulación informativa y narrativa (de acuerdo con los parámetros clásicos de los medios de comunicación, la estructura y el punto de vista narrativo del jugador), sobre la formulación ludonarrativa (elementos formales que integran los juegos) y, por último, sobre su localización de acuerdo con los rasgos que han definido a los newsgames hastala fecha.

\section{Construcción informativa y narrativa}

La ausencia de una presentación formal sobre el coronavirus en estos juegos permite afirmar que se dirigen a un público que ya conoce el efecto global de la pandemia y sus efectos. A partir de ahí se desarrollan dos direcciones informativas diferentes, de manera que, mientras People of the Pandemic ejemplifica el impacto de las medidas de confinamiento y hace hincapié en la responsabilidad individual que tiene cada individuo para frenar el contagio, Factitious 2020! Pandemic Edition y Covid-19 Trivia se centran en paliar y combatir las noticias falsas y la desinformación en torno a la covid-19. A pesar de lo reducido de la muestra, se comprueba por tanto que las propuestas lúdicas recogen dos de los principales vectores visibilizados en el contexto noticioso: la formación de una conciencia individual y colectiva para frenar los contagios (en positivo) y el alcance de la desinformación y las noticias falsas (en negativo). A la hora de abordar ambas dimensiones, cabe señalar que los juegos apuestan por dos fórmulas narrativas para desarrollar sus propuestas a nivel informativo: creación de reportajes subjetivos y aplicación de cuestionarios ludificados. 


\section{El reportaje subjetivo en People of the Pandemic}

People of the Pandemic plantea una apuesta narrativa vinculada al género periodístico definido en la categoría de "reportaje subjetivo", de modo que en la interfaz textual donde se ubica al usuario se recurre de forma continua al vocativo "tú". Con esta técnica de personalización, se involucra al lector y se consigue que la presentación de la información ofrecida durante el transcurso del juego resulte más estimulante.

La dirección presenta los objetivos de la simulación con apelaciones al jugador similares a las de los anuncios institucionales que los distintos países han utilizado para involucrar al individuo en su comunidad, hacerle sentir parte del colectivo ciudadano y frenar la propagación de la enfermedad. La finalidad no es otra que encontrar formas de satisfacer las necesidades individuales de las personas y, al mismo tiempo, limitar sus salidas de casa tanto como sea posible. En este escenario, la exposición a la información que se ofrece al jugador refleja el interés por ludificar un modelo que combina dos tipos de datos: los que miden la satisfacción de necesidades del jugador en su condición de individuo (comida, ejercicio y felicidad) y los que evalúan la condición sanitaria de la comunidad (casos de covid-19, infecciones por franja de edad, evolución, etcétera) ${ }^{\mathrm{ii}}$.

Imagen 1. Pantalla de juego en People of the Pandemic

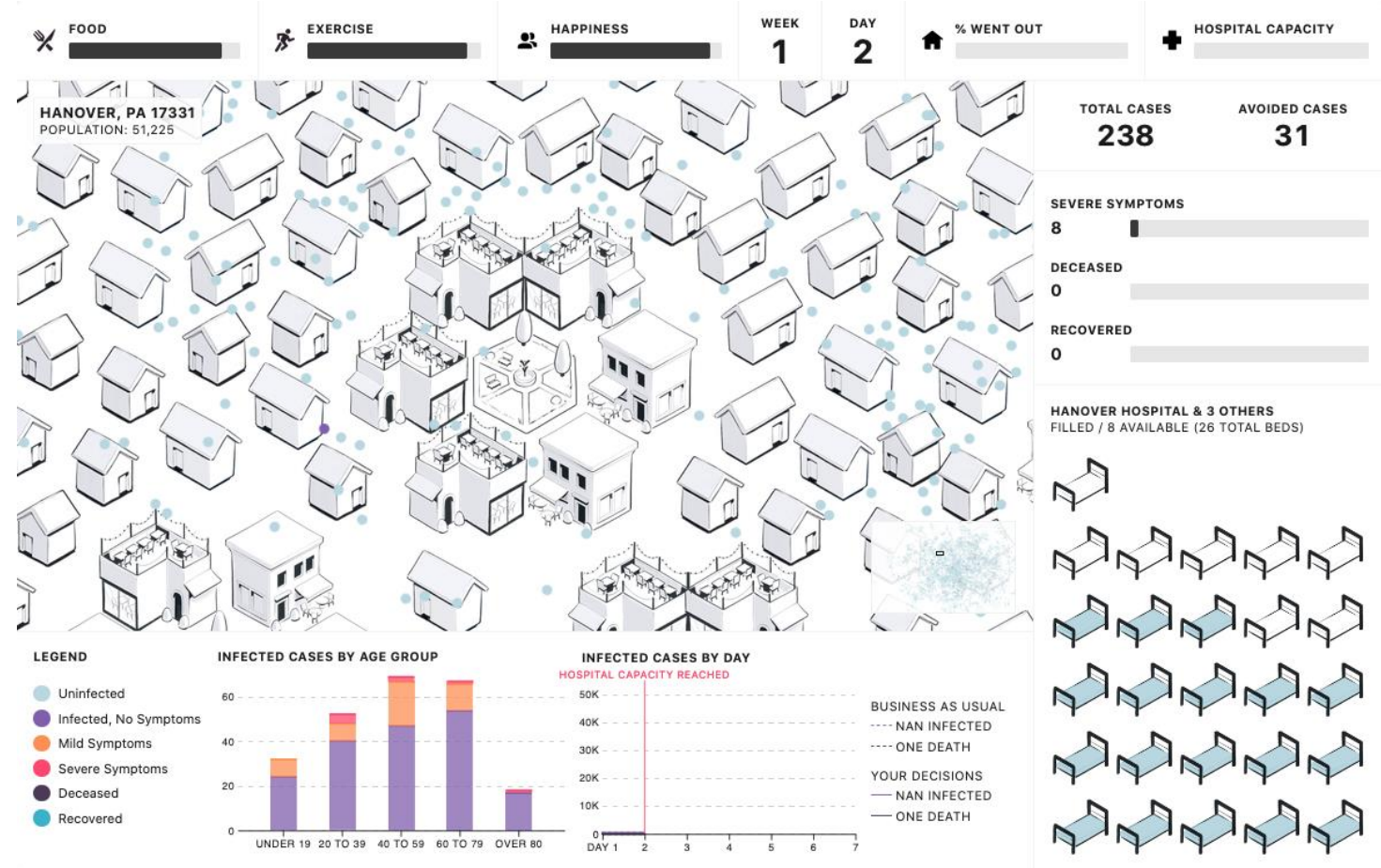

Fuente: People of the Pandemic (Wu \& Osserman, 2020).

\section{Cuestionarios ludificados en Factitious 2020! Pandemic Edition y Covid-19 Trivia}

Los otros dos juegos que componen la muestra de análisis impulsan una lógica que compromete los conocimientos de actualidad y sobre el virus que tiene el jugador, desde diferentes niveles de desarrollo. A este respecto es importante detallar que Covid19 Trivia se desarrolló durante una JAM (escenarios de desarrollo de videojuegos en 
intervalos cortos, de 24 o 48 horas), mientras que Factitious 2020! Pandemic Edition cuenta con un desarrollo más pulido. No en vano, esta última propuesta consiste en la actualización de un popular newsgame desarrollado por Augame Studio en 2017 y actualizado en 2020 con la temática de la covid-19.

Imagen 2. Presentación de Factitious 2020! Pandemic Edition; ejemplo de noticia para verificar; feedback en caso de respuesta correcta e incorrecta
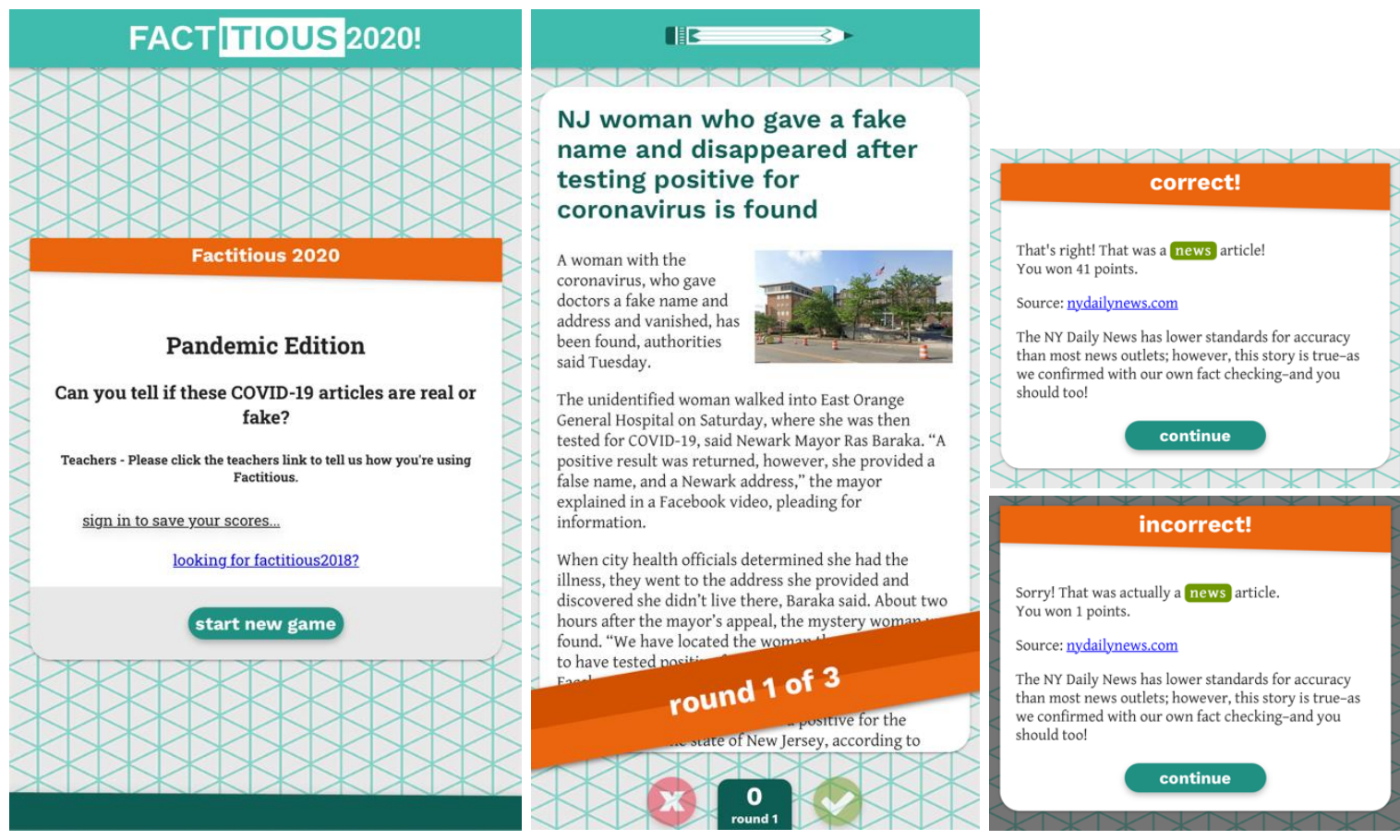

Fuente: Factitious 2020! Pandemic Edition (Augame Studio, 2017-20).

Las propuestas en ambos casos persiguen impulsar una alfabetización informativa sobre el coronavirus que posibilite paliar los efectos de la desinformación y las noticias falsas. Para ello se recurre a la estrategia de creación de un cuestionario ludificado en el que se incorporan opciones de difusión en redes sociales y otros elementos que se analizarán posteriormente y que, más que pretender evaluar los conocimientos del jugador, tratan de expandirlos.

En ambos formatos subyace un método periodístico basado en la incorporación de fuentes y referencias a todas las noticias que se citan (publicadas en algún medio o que se han producido a partir de casos reales), satisfaciendo así el interés de los newsgames por "anclarse" a la realidad a pesar de su naturaleza lúdica. Por último, la construcción de un discurso informativo en las partidas se traduce en resultados como el cumplimiento de objetivos acometidos con cierta rapidez (el creador de Covid1-9 Trivia señalaba unos tiempos de desarrollo de entre 12 y 24 horas), la actualización de estructuras ya existentes (Factitious 2020! Pandemic Edition) y la adaptación ludonarrativa de un modelo estadístico sobre la incidencia de los flujos de población en relación con la covid-19. 


\section{Conformación ludonarrativa del mensaje informativo}

El diseño del contenido informativo en el juego motiva al usuario para que se involucre de manera más activa en la simulación. Para ello se recurre a las estrategias que se analizan a continuación a partir de las tres dimensiones estéticas de los juegos digitales propuestas por Janet H. Murray en su obra Hamlet en la holocubierta: inmersión, actuación y transformación.

\section{Inmersión}

Janet H. Murray interpreta la inmersión en los juegos digitales como la implicación de "aprender a nadar, a hacer cosas que el nuevo entorno nos permite" (Murray, 1999, p. 111). Esta consideración establece que la construcción de una identidad virtual supone llevar a cabo la actuación a través de un avatar y tener la capacidad de crear patrones narrativos e interactivos que transportan al jugador, haciéndole entrar en un mundo diegético que vive como propio (Hartung, et al., 2016, p. 2).

Una de las principales estrategias de inmersión en los tres juegos analizados consiste en el uso recurrente del vocativo "tú". Curiosamente y a diferencia de lo que ocurre con otros newsgames, no se propone la construcción de una identidad alternativa para el jugador. La excepcionalidad, amplitud e intensidad de la pandemia hacen que no sea necesario contextualizar con abundantes detalles lo excepcional de la situación, ya que todos los jugadores conocen, de primera mano, lo que implica el coronavirus a nivel global.

La circunstancia expuesta condiciona el tipo de elecciones a las que el jugador se enfrenta en la partida. De hecho, el juego no coloca al usuario en circunstancias límite o exageradas, sino que se limita a plantear decisiones que se han vuelto cotidianas en 2020 (People of the Pandemic) o testa la capacidad de verificar noticias para discernir lo verdadero de lo falso (Factitious 2020! Pandemic Edition y Covid-19 Trivia).

\section{Actuación}

La capacidad de "poder llevar a cabo acciones significativas y ver los resultados de nuestras decisiones y elecciones" (Murray, 1999, p. 139) define el proceso de actuación en los juegos digitales. Esa preocupación porque se "reflejen" las consecuencias de cada acto está presente en la narrativa de los tres juegos que componen la muestra analizada. 


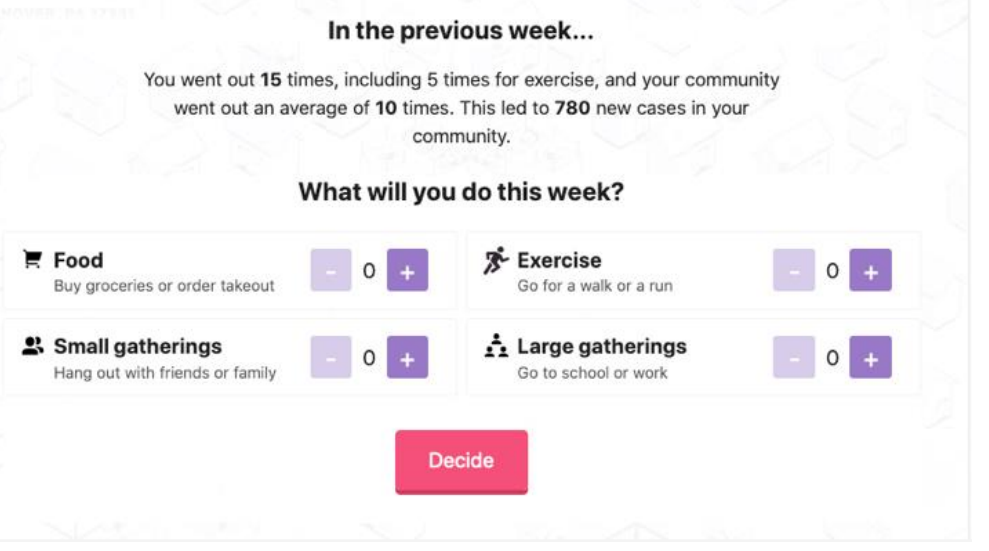

Fuente: People of the Pandemic (Wu \& Osserman, 2020).

En concreto, People of the Pandemic consulta al jugador cómo va a comportarse cada semana y qué tipo de decisiones piensa tomar en relación con actividades cotidianas en un contexto de confinamiento de la población. Estas elecciones que inicialmente son de carácter operativo (entre otras, hacer la compra, participar en reuniones sociales, practicar ejercicio o ir al trabajo) terminan convirtiéndose en disyuntivas morales cuando el jugador comprende el alcance de sus decisiones y comprueba cómo evolucionan las cifras de la pandemia.

En el caso de los juegos basados en cuestionarios, proponen un registro diferente. Su objetivo no es operativo ni moral, pues simplemente tratan de discernir la capacidad del jugador para diferenciar informaciones correctas de informaciones falsas. Para ello se recurre a la formulación de preguntas y a un sistema de puntuaciones que no sólo premian el acierto (tabla 2), sino también la capacidad de verificación por parte del usuario para "conformar la propuesta ideológica en la que el jugador participa al aceptar sus reglas" (Gómez-García \& Carrillo-Vera, 2020, p. 36).

Tabla 2. ¿Cómo funciona Factitious?

\begin{tabular}{|l|l|}
\hline Respuesta Correcta: +40 puntos \\
Respuesta Incorrecta: -10 puntos \\
Jugada rápida: hasta +7 puntos \\
Jugada lenta: hasta -5 puntos \\
Leer la fuente de la noticia: +2 puntos \\
Leer el texto del artículo: +1 punto \\
Fuente: "Cómo funciona" en Factitious 2020! Pandemic Edition.
\end{tabular}




\section{Transformación}

La capacidad de embaucar al jugador a través de una narrativa en primera persona y el compromiso que se genera con la propuesta de elegir el modo de actuar activa el proceso transformador de los juegos digitales que, en palabras de Janet H. Murray, se asienta en la idea de que "la narración es una fuerza poderosa de transformación personal [...] La representación tiene un poder transformador mayor que el de las historias narradas o interpretadas por otros, porque las asimilamos como experiencias propias" (Murray, 1999, pp. 182-183).

Los elementos anteriores constituyen los criterios a partir de los cuales se aprecia en los tres newsgames analizados el tipo de mensaje que se pretende trasladar. Como quiera que sea, determinar de manera precisa su alcance y la eficacia del discurso requiere, en última instancia, de un análisis de la recepción más amplio, que excede las pretensiones de esta investigación de enfoque eminentemente descriptivo.

\section{Discusión y conclusiones}

El objetivo básico de los newsgames inspirados en la temática de la covid-19 es permitir que el jugador alcance una conclusión que "ocurre cuando se comprende la estructura de una obra, aunque no se haya entendido su argumento" (Murray, 1999, p. 186). En este sentido, los planteamientos narrativos captan la atención de un jugador cuyo proceso de aprendizaje se completa en el momento en que detecta cómo funciona el juego, en la medida en que pretende explicar un vector de la realidad en torno al coronavirus: la importancia de la acción individual en materias de salud pública en People of the Pandemic, y la necesidad de verificar la información en Factitious 2020! Pandemic Edition y Covid-19 Trivia.

El análisis conjunto de las categorías apunta a la naturaleza pedagógica de los tres juegos digitales sobre los que, además, se intuye que las nuevas narrativas tienen el potencial de aproximarse más a los usuarios, dado que los propios jugadores detectan cómo la práctica lúdica tiene componentes pedagógicos que son capaces de identificar pese a ser desconocidos (Gómez-García et al., 2017, p. 52). De esta forma, explicar a través de los newsgames las formas de propagación del virus y sensibilizar a la población sobre los aspectos sociales de la covid-19 conforman, junto a otras dimensiones, un conjunto de actuaciones que han despertado el interés de la ciudadanía al alejarse de la sobreabundancia informativa tradicional que a menudo incrementa el umbral de incertidumbre de los ciudadanos (Mora-Rodríguez \& Melero-López, 2000).

La limitación de la muestra analizada en este trabajo obliga a ser cautos con la proyección de los resultados, pero ello no es óbice para presentar una imagen fija de algunos rasgos del entretenimiento informativo en la intersección entre los newsgames y la contribución al conocimiento sobre la alerta sanitaria. En cualquier caso, y a pesar de que se trata de un formato falto de consolidación en el ecosistema informativo (Bogost, 
2019), se concluye que la coyuntura de demanda de datos no ha sido aprovechada por parte de los mass media para presentar rasgos complejos de la pandemia bajo una fórmula que podría haber facilitado su comprensión para todos los públicos. Incluidos aquellos diarios como The Guardian o The New York Times que, en anteriores ocasiones, no dudaron en apostar por la gamificación.

Es difícil determinar si los motivos del escaso número de newsgames sobre el coronavirus en los medios se deben a que se trata de un tema arriesgado y polémico ante el riesgo de banalización de las circunstancias, aunque a este respecto cabe recordar que el formato sí se ha aplicado con éxito a asuntos tan delicados como un magnicidio -caso de JFK Reloaded (Burton, 2005, p. 90)- o a la crisis de los refugiados (Plewe \& Fürsich, 2018). De este modo y aunque no se puede descartar que la falta de oferta responda a una apuesta conservadora a la hora de abordar la temática sanitaria, predomina la hipótesis de que la sobrecarga de trabajo en las redacciones haya sido la verdadera causa de que los profesionales de la información hayan preferido opciones más consolidadas y automatizadas.

Al margen de la tímida apuesta por el formato protagonista de este artículo, concluimos dentro de él que el uso de newsgames en contextos donde se persigue la alfabetización informativa entraña más oportunidades que limitaciones, empezando por reducir el umbral de abstracción y confusión provocado por la pandemia. A este respecto, el siguiente paso en la investigación sería precisar los límites de unos recursos que, 15 años después de su aparición, siguen teniendo un prometedor futuro. Para seguir profundizando en él, como quiera que sea, tendremos que hacerlo al estilo de Mario Bros: en otro castillo.

\section{Bibliografía}

Andreu-Sánchez, C y Martín-Pascual, M.A. (2020). Fake images of the SARS-CoV-2 coronavirus in the communication of information at the beginning of the first covid-19 pandemic. El profesional de la información, v.29, n.3, e290309. https://doi.org/10.3145/epi.2020.may.09

Ballesteros Herencia, C. A. (2020). La propagación digital del coronavirus: Midiendo el engagement del entretenimiento en la red social emergente TikTok. Revista española de comunicación en salud, 171. https://doi.org/10.20318/recs.2020.5459

Bogost, I. (2020). Curiosity Journalism, or the First Decades of Newsgames. Convergence, 26(3), 572-576. https://doi.org/10.1177/1354856520918075

Bogost, I., Ferrari, S., \& Schweizer, B. (2010). Newsgames: Journalism at play. MIT Press.

Burton, J. (2005). News-game journalism: history, current use and possible futures. Australian Journal of Emerging Technologies and Society, 3(2), pp. 82-93. 
Carr, D. (2009). Textual analysis, Digital Games, Zombies. En DIGRA 2009 Conference: Breaking New Ground: Innovations in Games, Play, Practice and Theory.

Casero-Ripolles, A. (2020). Impact of covid-19 on the media system. Communicative and democratic consequences of news consumption during the outbreak. El Profesional de la Información, 29(2). https://doi.org/10.3145/epi.2020.mar.23

Casino, G. (2019). Retos y perspectivas en el ecosistema de la información biomédica. El profesional de la información, v.28, n.2, e280205. https://doi.org/10.3145/epi.2019.mar.05

Diviu-Miñarro, C., \& Cortiñas-Rovira, S. (2020). Cómo comunicar una pandemia a la sociedad: La visión de los profesionales. Estudio de caso de la covid-19 en el sur de Europa. El profesional de la información, v.29, n.5 e290512. https://doi.org/10.3145/epi.2020.sep.12

Dovey, J., \& Kennedy, H.W. (2006). Game Cultures. Computer Games as New Media. Open University Press.

Ferrer-Conill, R., \& Karlsson, M. (2016). The Gamification of Journalism. En Gangadharbatla, H., \& Davis, D.Z. (Eds.) Emerging Research and Trends in Gamification. AMIT.

Ferrer-Conill, R., Foxman, M., Jones, J., Sihvonen, T. \& Siitonen, M. (2020). Playful approaches to news engagement. Convergence: The International Journal of Research into New Media Technologies, 1-13

New York Times, The. (30, abril, 2020 - edición digital) How Coronavirus Mutates and Spreads. Jonathan Corum \& Carl Zimmer. Disponible en https://www.nytimes.com/interactive/2020/04/30/science/coronavirusmutations.html

Fundamentium, (2020) Las mejores infografías científicas y visualizaciones de datos sobre el coronavirus. Heber Longás. Disponible en https://fundamentium.com/articulo/las-mejores-infografias-cientificas-yvisualizaciones-de-datos-sobre-el-coronavirus/

García-Ortega, A., \& García-Avilés, J. (2018). Los newsgames como estrategia narrativa en el periodismo transmedia: propuesta de un modelo de análisis. Revista Mediterránea de Comunicación, 9(1), pp. 327-346. https://doi.org/10.14198/MEDCOM2018.9.1.19

Gil-Torres, A., Martín-Quevedo, J., Gómez-García, S., \& San José-De la Rosa, C. (2020). El coronavirus en el ecosistema de los dispositivos móviles: Creadores, discursos y recepción. Revista Latina, 78, 329-358. https://doi.org/10.4185/RLCS-20201480 
Gómez-García, S. \& Cabeza, J. (2016). El discurso informativo de los newsgames: el caso Bárcenas en los juegos para dispositivos móviles. Cuadernos.info, 38, 137148. https://doi.org/10.7764/cdi.38.593

Gómez-García, S., \& Carrillo-Vera, J.A. (2020). El discurso de los newsgames frente a las noticias falsas y la desinformación: cultura mediática y alfabetización digital. Revista Prisma Social, (30), pp. 22-46. Disponible en https://revistaprismasocial.es/article/view/3751

Gómez, Salvador. (2015). ¿Pueden los videojuegos cambiar el mundo? Logroño: UNIR Claves.

Gómez-García, S., Planells de la Maza, A., y Chicharro-Merayo, M. (2017). ¿Los alumnos quieren aprender con videojuegos? Lo que opinan sus usuarios del potencial educativo de este medio. Educar, 53(1), pp. 49-66. https://doi.org/10.5565/rev/educar.848

Hartung, F., Burke, M., Hagoort, P. \& Wilems, R.M. (2016). Taking perspective: Personal pronouns affect experiential aspects of literary reading. PloS ONE, 11(5), pp. 1-18. https://doi.org/10.1371/journal.pone.0157285

Kupferschmidt, K. (2020). Preprints bring 'firehose'of outbreak data. Science, v.367, n.6481, pp. 963-964. https://doi.org/10.1126/science.367.6481.963

Mañas-Viniegra, L., Zamith, F., \& López-Cepeda, I. (2019). Newsgames, más allá de la información y la reputación corporativa: el caso The Uber Game. En TorresToukoumidis, A. y Romero-Rodríguez, L.M. (Eds.) Juegos y Sociedad. Desde la interacción a la inmersión para el cambio social.

McLuhan, M. (1996). Comprender los medios de comunicación: las extensiones del ser humano. Paidós.

Masip, P., Aran-Ramspott, S., Ruiz-Caballero, C., Suau, J., Almenar, E., \& PuertasGraell, D. (2020). Consumo informativo y cobertura mediática durante el confinamiento por el covid-19: Sobreinformación, sesgo ideológico y sensacionalismo. El Profesional de la Información, 29(3). https://doi.org/10.3145/epi.2020.may.12

Meneses-Fernández, M. D., \& Santana-Hernández, J. D. (2020). Construcción periodística de la mala praxis científica: análisis en diarios digitales. Revista Prisma Social (31), 40-63. Recuperado dehttps://revistaprismasocial.es/article/view/3853

Meier, K. (2018). Journalism meets games: Newsgames as a new digital genre. Theory, boundaries, utilization. Journal of Applied Journalism y Media Studies, 7(2), pp. 429-444.https://doi.org/10.1386/ajms.7.2.429_1 
Monjas Eleta, M., Rodríguez Holguín, A. y Gil-Torres, A. (2020). La covid-19 en las portadas de los diarios de difusión nacional en España. Revista de Comunicación y Salud, 10(2), pp. 265-286. https://doi.org/10.35669/rcys.2020.10(2).265-286

Mora-Rodríguez, A., \& Melero-López, I. (2021). News consumption and risk perception of covid-19 in Spain. Comunicar, 66. https://doi.org/10.3916/C66-2021-06

Murray, J. (1999). Hamlet en la holocubierta: el futuro de la narrativa en el ciberespacio. Paidós.

New York Times, The. (30, abril, 2020 - edición digital) How Coronavirus Mutates and Spreads. Jonathan Corum \& Carl Zimmer. Disponible en https://www.nytimes.com/interactive/2020/04/30/science/coronavirusmutations.html

Plewe, C. \& Fürsich, E. (2018). Are newsgames better journalism? Empathy, information and representation in games on refugees and migrants. Journalism Studies, 19(16), pp. 2470-2487. https://doi.org/10.1080/1461670X.2017.1351884

País, El. (24, octubre, 2020 - edición digital) Un salón, un bar y una clase: así contagia el coronavirus en el aire. Disponible en https://elpais.com/ciencia/2020-10-24/un$\underline{\text { salon-un-bar-y-una-clase-asi-contagia-el-coronavirus-en-el-aire.html }}$

Rodero, E. (2020). La radio: El medio que mejor se comporta en las crisis. Hábitos de escucha, consumo y percepción de los oyentes de radio durante el confinamiento por el covid-19. El Profesional de la Información, 29(3). https://doi.org/10.3145/epi.2020.may.06

Romero-Rodríguez, L.M., \& Rodríguez-Hidalgo, C. (2019). "Desinformación y posverdad en los medios digitales: del astroturfing al click baiting". En L.M. Romero-Rodríguez, \& D.E. Rivera-Rogel (Eds.), La comunicación en el escenario digital. Actualidad, retos y prospectivas, (pp. 379-407) Pearson. https://doi.org/10.16921/chasqui.v0i140.4033

Salles, M. L. M., \& Dall'Orto, F. C. (2020). The Impact of Covid-19 on the Journalistic Narrative: The Production of Content from Folha de S. Paulo. Revista Observatório, 6(3), a9en. https://doi.org/10.20873/uft.2447-4266.2020v6n3a9en

Sicart, M. (2008). Newsgames: Theory and Design. En S. M. Stevens \& S. J. Saldamarco (Eds.), Entertainment Computing-ICEC 2008 (Vol. 5309, pp. 27-33). Springer Berlin Heidelberg. https://doi.org/10.1007/978-3-540-89222-9_4

Sidorenko-Bautista, P., Herranz de la Casa, J.M. \& Cantero de Julián, J.I. (2020). Use of New Narratives for covid-19 Reporting: From $360^{\circ}$ Videos to Ephemeral TikTok Videos in Online Media. Tipodos v.2, n.47, 105-122. Disponible en http://www.tripodos.com/index.php/Facultat_Comunicacio_Blanquerna/article/v iew/806 
Torres-Salinas, D. (2020). Ritmo de crecimiento diario de la producción científica sobre covid-19. Análisis en bases de datos y repositorios en acceso abierto. El Profesional de la Información, 29(2). https://doi.org/10.3145/epi.2020.mar.15

Usher, N. (2014). Making news at The New York Times. Michigan: University of Michigan Press.

Vicente-Fernández, P., Vinader-Segura, R., \& Puebla-Martínez, B. (2020). Padres ante el desafío educativo en situación de confinamiento: análisis comparativo entre Educación Infantil y Educación Primaria. Revista De Estilos De Aprendizaje, 13(Especial), pp. 56-67. Disponible en http://revistaestilosdeaprendizaje.com/article/view/2155

Villena-Alarcón, E., \& Caballero-Galeote, L. (2020). Covid-19 Media Coverage on Spanish Public TV. Tipodos v.2, n.47, pp. 103-126. Disponible en: http://www.tripodos.com/index.php/Facultat_Comunicacio_Blanquerna/article/v iew/818/837

Vobič, I., Dvoršak, L., \& Vtič, M. (2014). Digital games and journalism: A case-study of slovenian political weekly'snewsgame- mladina's fojba 2000. Teorija in Praksa, 51(1), pp. 123-142.

\section{Notas}

\footnotetext{
${ }^{\mathrm{i}}$ Este manuscrito es resultado del Proyecto de Investigación I+D+i «Politainment en el entorno de la posverdad: nuevas narrativas, clickbait y gamificación» (CSO2017-84472-R), subvencionado por el Ministerio de Economía, Industria y Competitividad del Gobierno de España.

ii La metodología que subyace en el modelo estadístico está reflejada en el propio juego y se puede consultar aquí: https://observablehq.com/@osserman/people-of-the-pandemic-simulationmethodology
} 